\title{
Neurogenic markers of the inflammatory process in atopic dermatitis: relation to the severity and pruritus
}

\author{
Ewa Teresiak-Mikołajczak, Magdalena Czarnecka-Operacz, Dorota Jenerowicz, Wojciech Silny
}

Department of Dermatology, Poznan University of Medical Sciences, Poznan, Poland

Head of Department: Prof. Zygmunt Adamski MD, PhD

Postep Derm Alergol 2013; XXX, 5: 286-292

DOI: $10.5114 /$ pdia.2013.38357

\begin{abstract}
Introduction: Atopic dermatitis (AD) is a chronic and relapsing inflammatory skin disease, characterized by eczematous skin lesions and intensive pruritus. Recent studies have shed light on the role of the nervous system in the pathogenesis of AD. It can influence the course of the disease through an altered pattern of cutaneous innervation and abnormal expression of neuropeptides in the lesional skin.

Aim: The aim of the study was to evaluate plasma concentrations of the nerve growth factor (NGF), substance $P$ (SP) and vasoactive intestinal peptide (VIP) in AD patients in comparison to two control groups (healthy volunteers and patients suffering from psoriasis). Correlations between plasma levels of evaluated parameters, severity of the disease and selected clinical parameters (skin prick tests, total and antigen specific lgE levels) were also analysed. Material and methods: Seventy-five patients with AD, 40 patients with psoriasis and 40 healthy volunteers were included into the study. Patients with AD included 52 persons suffering from an extrinsic and 23 from an intrinsic type of the disease. The severity of skin lesions was assessed with SCORAD index. Pruritus was evaluated on the basis of the questionnaire assessing the extent, frequency and intensity of pruritus. Commercial enzyme-linked immunosorbent assays (SP, NGF: R\&D Systems; and VIP: Phoenix Pharmaceuticals) were used to assess the neuropeptide and NGF plasma levels.

Results: Nerve growth factor and VIP plasma concentrations were significantly higher in AD patients compared to psoriatic patients and healthy subjects. Substance $P$ plasma concentrations were elevated in the extrinsic type of $\mathrm{AD}$ and psoriasis comparing to healthy volunteers. There were no statistically significant differences in NGF, SP and VIP plasma concentrations between the extrinsic and intrinsic type of AD. There was also no correlation between plasma levels of evaluated parameters (NGF, SP, VIP) and SCORAD index in both types of AD. However, plasma SP concentration correlated with intensity of pruritus in AD patients. Plasma VIP concentrations correlated with intensity of pruritus in the intrinsic type of $A D$ and with IgE-mediated sensitization to moulds in the extrinsic type of disease.

Conclusions: Our findings confirm that NGF and VIP play a prominent role in atopic inflammatory reactions and may serve as good alternative biomarkers of AD. The results of this study also suggest a similar important role of neuroimmune interactions in both variants of AD. Increased SP plasma concentrations in both AD and psoriasis point to its possible role in modulating immune-mediated inflammation in different chronic inflammatory skin diseases. Moreover, SP and VIP seem to influence the course of AD by increasing pruritus, whereas an elevated plasma VIP level in $A D$ patients may be related to a risk of developing IgE-mediated sensitization to certain airborne allergens.
\end{abstract}

Key words: atopic dermatitis, neuropeptides, intrinsic type, extrinsic type.

\section{Introduction}

Atopic dermatitis (AD) is a chronic and relapsing inflammatory skin disease, characterized by eczematous skin lesions and intensive pruritus. The etiology of AD is very complex and multifactorial with interactions between genetic, environmental and immunologic factors [1, 2]. Two distinctive types of disease have been distinguished based on IgE sensitization: an extrinsic and intrinsic type of AD. According to the European Academy of Allergology and Clinical Immunology classification, nonallergic and aller-

Address for correspondence: Ewa Teresiak-Mikołajczak MD, PhD, Department of Dermatology, Poznan University of Medical Sciences, 49 Przybyszewskiego St, 60-355 Poznan, Poland, phone: +48 601957 851, e-mail: ewa.teresiak@wp.pl Received: 10.05.2013, accepted: 20.08.2013. 
gic types of atopic eczema/dermatitis syndrome (AEDS) have been delineated [3]. Both types share the same clinical features, but demonstrate different immunological parameters. In contrast to the extrinsic type, patients with the intrinsic form of disease have a normal total lgE level and negative serum allergen-specific IgE, as well as a decreased IL-4 and IL-13 expression in skin T cells $[1,4]$. Recent studies have shed light on the role of the nervous system in pathogenesis of AD [5, 6]. It can influence the course of the disease through an altered pattern of cutaneous innervation and abnormal expression of neuropeptides in lesional skin $[7,8]$. Neuropeptides exert many actions which contribute to the development of $A D$ and influence the course of the disease [6]. Substance P (SP) and vasoactive intestinal peptide (VIP) are potent vasodilators, cause degranulation of mast cells and induce chemotaxis of neutrophils and lymphocytes. Moreover, neuropeptides regulate cytokines release, influence activity of immunological cells and lower the itch threshold [9-13].

Increasing evidence suggests that neurotrophins such as the nerve growth factor (NGF) may also be involved in the pathogenesis of $A D$ and may regulate the development of the disease [13]. The nerve growth factor and its receptors play an important role in the development of the peripheral nervous system including cutaneous nerves. Its effects include neuronal growth, differentiation and regeneration of injured or diseased nerves. It is suggested that NGF determines skin innervation density and contributes to neurohyperplasia in $\mathrm{AD}[14,15]$. Moreover, an increased neurotrophin receptor expression and neurotrophins functional activity lowers the itch thresholds in a mouse AD model $[16,17]$. High concentrations of plasma NGF is considered a risk factor for developing the disease. Evidence suggests a correlation between an increased cord or maternal NGF level and risk of developing AD before the age of 2 [18].

Recent studies have also shown that psychological stress induces degranulation of dermal mast cells and enhances the number of SP-positive nerve fibers in mice $[19,20]$. Moreover, increasing evidence suggests that stress triggers mast cell degranulation via SP and VIP in patients with AD thus exacerbating the disease [20, 21]. In addition, Staphylococcal enterotoxins enhance neurogenic inflammation in animals. Immunofluorescence staining revealed increased innervation of sensory C-fibers and expression of SP receptors in rat skin, probably due to upregulated production of NGF after exposure to Staphylococcal enterotoxins [22, 23].

\section{Aim}

The aim of the study was to evaluate plasma concentrations of NGF, SP and VIP in AD patients in comparison to two control groups (healthy volunteers and psoriatic patients). A comparison of neuropeptide plasma concentrations between two types of AD (intrinsic and extrinsic type) was also performed. The additional aim was to evaluate correlations between plasma levels of measured parameters and severity of the disease. In addition, an assessment of correlation between plasma levels of NGF, SP and VIP and selected clinical parameters (skin prick tests, total and antigen specific lgE levels) was performed.

\section{Material and methods}

An ethical approval for our study was obtained from the ethics committee of the Poznan University of Medical Sciences. A full explanation of the procedure was given to all participants, and their written consent was obtained before testing. Seventy five patients with $A D, 40$ patients with psoriasis vulgaris and 40 healthy volunteers were included in the study. Patients with AD included 52 persons suffering from an extrinsic and 23 from an intrinsic type of the disease. Patients enrolled into the extrinsic type presented IgE-mediated sensitization to common food and/or aeroallergens (positive results of skin prick tests and/or elevated total and antigen-specific IgE serum levels). This group comprised 21 males (40.4\%) and 31 females (59.6\%) and mean age was $20.5 \pm 12.7$ years. Patients with the intrinsic type of AD included 11 males (47.8\%) and 12 females (52.2\%), with no IgE-mediated sensitization to food and/ or aeroallergens and mean age was $15.8 \pm 8$. 8 years. In addition, there were 40 nonatopic healthy volunteers (mean age $33.7 \pm 11.8$ years) and 41 psoriatic patients (mean age $38.5 \pm 13$ years).

The severity of skin lesions was assessed with SCORAD index (Severity Scoring of Atopic Dermatitis). Pruritus was evaluated on the basis of the questionnaire assessing the extent, frequency and intensity of pruritus as well as intensity of sleep disturbances caused by itch. In the AD group, skin prick tests with airborne and food allergens (Allergopharma, Reinbeck, Germany) were performed. Total and antigen-specific IgE levels were evaluated with the use of fluoroenzyme immunoassay.

Commercial enzyme-linked immunosorbent assays (SP, NGF: R\&D Systems; and VIP: Phoenix Pharmaceuticals) were used to assess the neuropeptide and NGF plasma levels according to the manufacturer's recommendations. All allergological diagnostic procedures were performed at the Allergic Disease Diagnostic Center at the Department of Dermatology, Medical University of Poznan.

\section{Statistical analysis}

All data were analyzed statistically by Mann-Whitney $U$ test, Kruskal-Wallis test and Student's t-tests for dependent and independent variables. The correlations between parameters were assessed with the Spearman rank test. Results were considered statistically significant when $p<0.05$. 


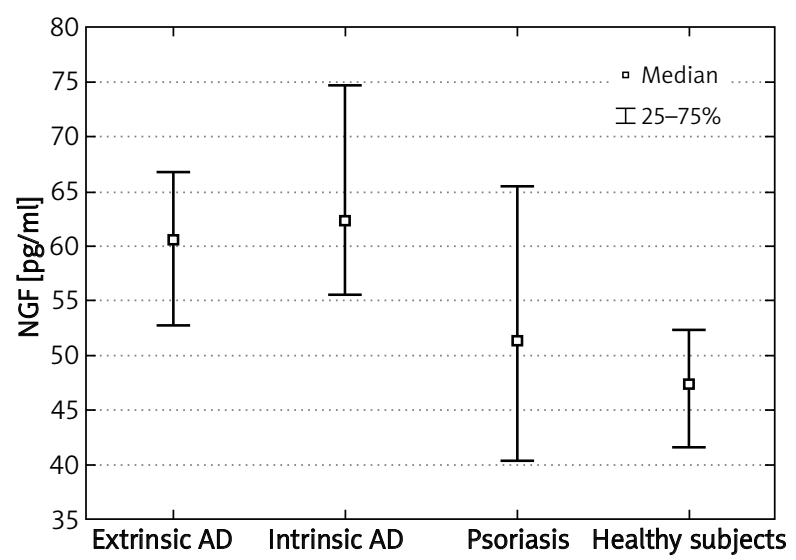

Figure 1. Significantly increased plasma concentrations of NGF in patients with extrinsic and intrinsic types of $A D$ compared to healthy subjects and patients with psoriasis

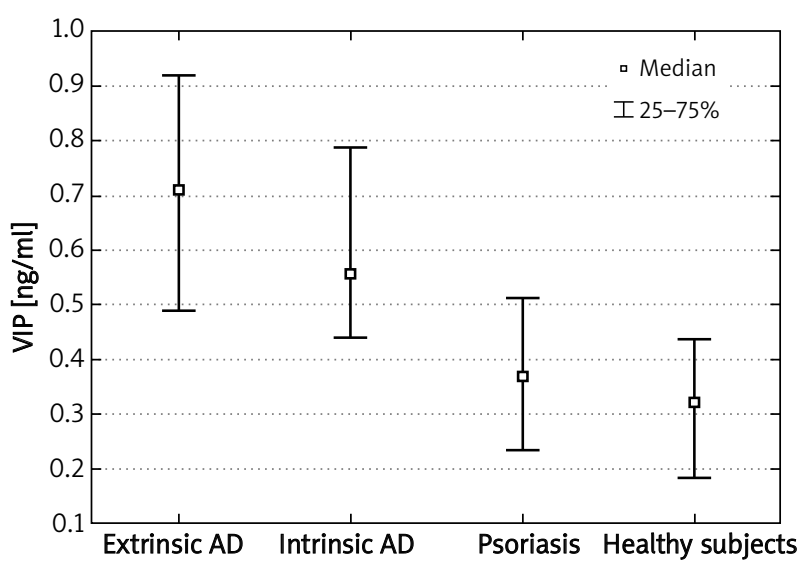

Figure 2. Significantly increased plasma concentrations of VIP in patients with extrinsic and intrinsic types of AD compared to healthy subjects and patients with psoriasis

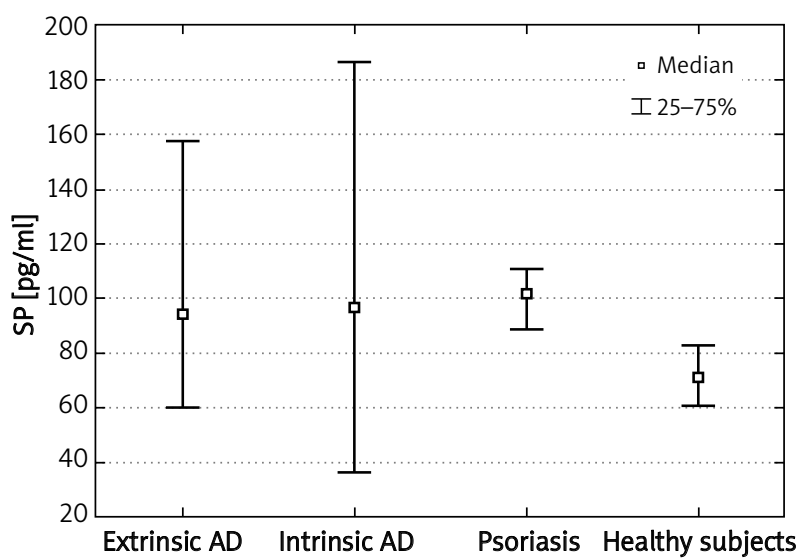

Figure 3. Significantly increased plasma concentrations of $S P$ in patients with the extrinsic type of $A D$ and patients with psoriasis compared to healthy subjects

\section{Results}

\section{Higher plasma levels of NGF and VIP in atopic dermatitis patients compared to psoriatic patients and healthy subjects}

We were able to detect NGF, SP and VIP concentrations in all evaluated subjects. Nerve growth factor plasma concentrations in patients suffering from extrinsic or intrinsic types of $A D$ were significantly higher comparing to healthy subjects $(p<0.001 ; p<0.0000001$, respectively). In addition, plasma NGF levels were higher in both phenotypes of AD compared to psoriasis ( $p<0.03 ; p<0.05$, respectively) (Figure 1). Vasoactive intestinal peptide concentrations were also significantly higher in patients with extrinsic or intrinsic types of $A D$ comparing to nonatopic healthy subjects $(p<0.001 ; p<0.0000001$, respectively). Moreover, VIP plasma levels in AD patients were also higher in comparison to patients suffering from psoriasis $(p<0.000001$ for the extrinsic and $p<0.05$ for the intrinsic type of $A D$ ) (Figure 2). There were no statistically significant differences in NGF and VIP plasma concentrations between the extrinsic and intrinsic types of AD.

\section{Substance $P$ plasma concentrations are elevated in the extrinsic type of $A D$ and psoriasis}

Substance P plasma concentrations were statistically higher in the extrinsic type of AD comparing to healthy subjects $(p<0.01)$. There were no significant differences in plasma SP levels between the intrinsic type of $A D$ and healthy volunteers. In addition we did not notice any significant differences in plasma SP levels between either extrinsic and intrinsic type of $A D$ or both phenotypes of $A D$ and patients suffering from psoriasis. However, SP plasma levels were significantly higher in patients with psoriasis compared to healthy subjects $(p<0.0001)$ (Figure 3$)$.

Results of plasma levels of evaluated neuropeptides (median, first and third quartile values) are presented in Table 1.

\section{Plasma VIP concentrations correlate with intensity of pruritus in the intrinsic type of $A D$ and with IgE-mediated sensitization to moulds in the extrinsic type of disease}

We did not record any significant correlations between plasma levels of evaluated parameters (NGF, SP, VIP) and SCORAD index in both types of AD. However, in patients with the intrinsic type of AD, plasma VIP levels correlated with intensity of pruritus (Figure 4). Another finding was a significant correlation of VIP plasma concentrations and IgE-mediated sensitization to moulds Cladosporium herbarum $(r=0.688 ; p=0.019)$ as well as Alternaria alternata $(r=0.8233 ; p=0.022)$. We also obtained a significant relationship between plasma levels of SP and severity of itch in the global population of AD patients (combined extrinsic and intrinsic types, $r=0.234893$; $p=0.042$ ) (Figure 5). There was no statistically significant 
Table 1. Plasma concentrations of NGF, SP and VIP in patients with AD and in control groups

\begin{tabular}{lccccccccc}
\hline Patients & \multicolumn{3}{c}{ NGF $[\mathrm{pg} / \mathrm{ml}]$} & \multicolumn{3}{c}{ SP $[\mathrm{pg} / \mathrm{ml}]$} & \multicolumn{3}{c}{ VIP [ng/ml] } \\
\cline { 2 - 11 } & Q25 & Median & Q75 & Q25 & Median & Q75 & Q25 & Median & Q75 \\
\hline Extrinsic type of AD & 52.7 & 60.7 & 66.8 & 60.4 & 94.5 & 157.3 & 0.50 & 0.71 & 0.92 \\
\hline Intrinsic type of AD & 55.6 & 62.4 & 74.8 & 36.5 & 97.0 & 185.9 & 0.44 & 0.56 & 0.79 \\
\hline Psoriasis & 40.4 & 51.4 & 65.5 & 88.4 & 101.5 & 110.6 & 0.23 & 0.37 & 0.51 \\
\hline Healthy subjects & 41.6 & 47.5 & 52.3 & 60.8 & 71.1 & 82.7 & 0.19 & 0.32 & 0.43 \\
\hline
\end{tabular}

correlation between plasma levels of other evaluated parameters (NGF, SP) and IgE-mediated sensitization to analyzed airborne and food allergens. Moreover, there was no significant correlation between analyzed neuropeptides and total IgE concentration in atopic individuals.

\section{Discussion}

It has been suggested that an altered pattern of cutaneous innervation and abnormal expression of neuropeptides in the lesional skin play an important role in the pathogenesis of AD. Studies evaluating neuropeptides levels in the lesional skin of AD patients showed increased concentrations of VIP and decreased concentrations of SP compared to healthy controls [24, 25]. Moreover, in AD patients intradermally injected SP produce in general a weaker inflammatory response comparing to healthy volunteers. This is probably resulting from a decreased number of SP receptors or their hyporesponsiveness due to excessive SP release by cutaneous nerve fibers. Constant release of SP may in turn result in desensitization of nerve fibers and a decreased SP concentration in the lesional skin $[26,27]$. On the other hand, increased density of SP-positive and unchanged density of VIP-positive nerve fibers was observed immunohistochemically in the atopic lesional skin compared to control groups [26-28]. Higher density and activity of nerve fibers within the papillary dermis and epidermis in AD is probably due to increased NGF production and release by basal keratinocytes. Recent studies confirmed strong immunoreactivity of NGF-positive cells and its receptors in the basal layer of epidermis and upper dermis in the course of $A D[15,28]$. Moreover, a higher expression of NGF receptors on peripheral blood eosinophils is observed in patients with AD comparing to healthy subjects [29]. Recent findings suggest that targeting NGFinduced epidermal hyperinnervation results in reduction in the density of SP-positive cutaneous nerve fibers and the number of inflammatory cells in atopic skin followed by the suppression of hypersensitivity to itch and improvement of the skin barrier function in an animal model of $A D$ $[22,30]$

The results of our study demonstrate that patients suffering from $A D$ have significantly higher plasma levels of NGF and VIP compared to psoriatic patients and healthy subjects whereas SP plasma concentrations are elevated in both the extrinsic type of AD and psoriasis. Moreover, our results indicate that plasma VIP concentrations correlate with selected clinical features of the disease such as intensity of pruritus in the intrinsic type of $A D$ and with IgE-mediated sensitization to moulds in the extrinsic type of disease. The data concerning neuropeptide plasma concentrations in $A D$ are often very conflicting. Raap et al. also

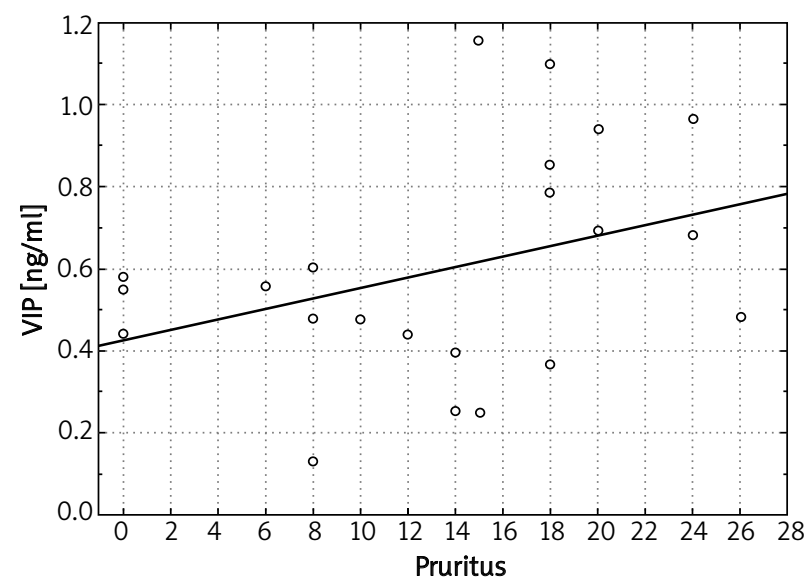

Figure 4. Significant correlation between plasma VIP concentrations and pruritus severity in the intrinsic type of $A D$

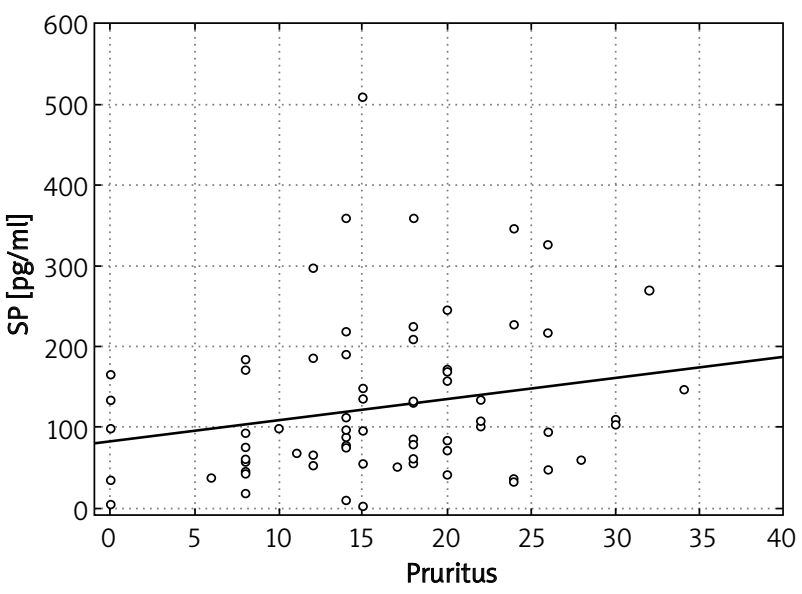

Figure 5. Significant correlation between plasma SP concentrations and pruritus severity in the whole group of $A D$ patients (both types of disease) 
observed a higher NGF plasma level in patients with both extrinsic and intrinsic types of AD compared to patients with psoriasis and healthy volunteers [29]. Toyoda et al. found statistically higher NGF and SP plasma concentrations in patients with AD compared to healthy subjects and a strong correlation between plasma levels of measured parameters and disease activity [13]. This is in agreement with our results, however we failed to find the relationship between NGF and SP plasma levels and total IgE, antigenspecific lgE or severity of the disease. Findings of other studies also confirm that NGF or neuropeptides plasma/serum levels are not clinical markers that are positively correlated with severity of $A D[5,31,32]$. It is known that NGF expression is increased in the skin of patients with $A D$ and is related to the disease exacerbation $[15,33]$. No correlation between plasma concentration of NGF and disease activity may be due to the fact that the plasma level does not completely reflect its level within the skin. The concentration of NGF in the horny layer (i.e. horn NGF) increases both in the non-lesional and lesional skin of AD patients in comparison to non-lesional skin of healthy subjects and correlates to some extent with disease activity [22]. Recent experiments revealed an increased NGF concentration within the inflamed skin during stress-induced exacerbation in an animal AD model, while the plasma NGF level remains unchanged [34]. Nerve growth factor is now regarded as a main factor regulating interactions between neuronal and immune cells and one of the most important mediators lowering the itch threshold. Emotional stress enhances the plasma NGF level and influences the NGF receptors' expression in lymphocytes [35]. Moreover, recent findings suggest an association of emotional stress and anxiety with the induction and enhancement of pruritus through NGF in AD patients [9]. Wang et al. showed a significant correlation between self-reported stress and plasma NGF in pregnant women [18].

Another interesting finding for us was a significant correlation between VIP plasma concentrations and IgE-mediated sensitization to moulds such as Cladosporium herbarum and Alternaria alternate. Therefore, we concluded that an increased plasma VIP level may be related to the intensity of allergic hypersensitivity and certain risk of developing IgE-mediated sensitization to selected airborne allergens. This is in agreement with findings of Herberth et al., who showed that an increased plasma VIP level correlates with an increased concentration of antigen-specific IgE to certain food allergens in children [36-39]. Recent studies confirm the association of VIP with Th1/Th2 balance and allergic sensitization in children [32]. Vasoactive intestinal peptide is a major determinant of the T-cells effector phenotype differentiation. It selectively increases the number and activation of Th2 cells over Th1 by promotion of antigen-dependent differentiation to Th2 cells [40]. On the other hand, there is increasing evidence that only Th2 cells produce VIP, thus VIP is considered as Th2 cytokine and its elevated plasma concentration may be regarded as an allergy risk factor [27, 41].

There is an increasing body of evidence that neuromediators such as neurotrophins or neuropeptides may play an important role in the intrinsic type of AD. Raap et al. found a significant correlation between disease activity and BDNF (brain derived neurotrophic factor) in the intrinsic type of AD. We did not record significant differences between the extrinsic and intrinsic type of $A D$ with regard to NGF and evaluated neuropeptides plasma concentrations. This is in agreement with findings of other authors who also failed to observe significant differences in plasma concentrations of NGF and SP between both types of disease. Therefore, a prominent role of neuroimmune interactions in both the extrinsic and intrinsic type of AD should be suggested. Both types share the same clinical features, but demonstrate different immunological parameters. The intrinsic type is associated with lower IL-4 and IL-13 production than the extrinsic type of the disease $[29,42]$. In addition, exposure to psychological stress enhances allergen-specific responses, increases IL-4 and IL-13 production via SP, VIP and NGF release in AD patients skewing of the cytokine pattern towards the Th2 type [20]. Therefore, it is possible that neuropeptides are responsible for stress-related exacerbations and alterations of the immunological system in both allergic and non-allergic skin inflammation in the course of AD.

\section{Conclusions}

Our findings confirm the important role of NGF and evaluated neuropeptides in the pathogenesis of AD. We assessed significantly higher plasma levels of NGF and VIP in AD patients compared to psoriatic patients and healthy subjects whereas SP plasma concentrations were elevated in both the extrinsic type of AD and psoriasis. This underlines that NGF and VIP play a prominent role in atopic inflammatory reactions and may serve as good alternative biomarkers of AD. Results of this study also suggest a similar prominent role of neuroimmune interactions in both variants of AD. On the other hand, SP plasma concentrations are elevated in both $A D$ and psoriasis pointing out to its possible role in modulating immune-mediated inflammation in the case of various chronic inflammatory skin diseases. In this regard, increased plasma concentrations of SP may be responsible for the chronic character and tendency for relapses of skin inflammatory diseases. Moreover, neuropeptides seem to influence the course of AD by increasing pruritus probably via lowering the itch threshold of cutaneous nerve fibers. In addition, elevated plasma VIP levels in AD patients may be related to the risk of developing lgEmediated sensitization to selected airborne allergens. Although plasma concentrations of neuromediators do not mirror the disease activity in AD, neuropeptides and neurotrophins released locally in the skin reflect the inflammation intensity and correlate with activity of the process. 
In conclusion, NGF and VIP plasma concentrations increase selectively in AD whereas the evaluation of SP and VIP plasma concentrations may be useful in assessing the intensity of itch in $A D$ patients.

\section{References}

1. Leung DY, Boguniewicz M, Howell MD, et al. New insight into atopic dermatitis. J Clin Invest 2004; 113: 651-7.

2. Żukiewicz-Sobczak W, Krasowska E, Zwoliński J, et al. Allergic diseases - current state of knowledge. Postep Derm Alergol 2012; 29: 451-5.

3. Johansson SG, Hourihane JO, Bousquet J, et al. A revised nomenclature for allergy. An EAACI position statement from the EACCI nomenclature task force. Allergy 2001; 56: 813-24.

4. Akdis CA, Akdis M. Immunological differences between intrinsic and extrinsic types of atopic dermatitis. Clin Exp Allergy 2003; 33: 1618-21.

5. Schulte-Herbrüggen O, Fölster-Holst R, von Elstermann M. Clinical relevance of nerve growth factor serum levels in patients with atopic dermatitis and psoriasis. Int Arch Allergy Immunol 2007; 144: 211-6.

6. Salomon J, Baran E. The role of selected neuropeptides in pathogenesis of atopic dermatitis. JEADV 2008; 22: 223-8.

7. Pincelli C, Fantini F, Massimi P, et al. Neuropeptides in skin from patients with atopic dermatitis: an immunohistochemical study. Br J Dermatol 1990; 122: 745-50.

8. Tobin D, Nabarro G, Baart de la Faille H, et al. Increased num ber of immunoreactive nerve fibers in atopic dermatitis. J Allergy Clin Immunol 1992; 90: 613-22.

9. Sang Ho OH, Byung Gi BAE, Chang Ook Park, et al. Association of stress with symptoms of atopic dermatitis. Acta Derm Venereol 2010; 90: 582-8

10. Lambrecht BN. Immunologists getting nervous: neuropeptides, dendritic cells and T cell activation. Respir Res 2001; 2: $133-8$.

11. Yoshiki I, Takei H, Matsumoto C, et al. Adminstration of substance $P$ during a primary immune response amplifies the secondary immune response via a long-lasting effect on CD8+ T lymphocytes. Arch Dermatol Res 2007; 299: 345-51.

12. O'Connor TM, O'Connell J, O'Brien DI, et al. The role of sub stance $P$ in inflammatory disease. J Cell Physiol 2004; 201: 167-80.

13. Toyoda M, Nakamura M, Makino T, et al. Nerve growth factor and substance $P$ are useful plasma marker of disease activity in atopic patients. Br J Dermatol 2002; 147: 71-9.

14. Nockher WA, Renz H. Neurotrophins in allergic diseases: from neuronal growth factor to intercellular signaling molecules. J Allergy Clin Immunol 2006; 117: 583-9.

15. Dou YC, Hagstromer L, Emtestam L. Increased nerve growth factor and its receptors in atopic dermatitis: an immunohistochemical study. Arch Dermatol Res 2006; 298: 31-7.

16. Ständer S, Steinhoff M. Pathophysiology of pruritus in atopic dermatitis: an overview. Exp Dermatol 2002; 11: 12-24.

17. Tanaka A, Matsuda H. Evaluation of itch by using NC/Nga Tnd mice: a model of human atopic dermatitis. J Biomed Biotech 2011; 2011: 790436.

18. Wang IJ, Hsieh WS, Guo YL. Neuro-mediators as predictors of paediatric atopic dermatitis. Clin Exp Allergy 2008; 38: 1302-8.

19. Kawana S, Liang Z, Nagano M, et al. Role of substance P in stress-derived degranulation of dermal mast cells in mice. J Dermatol Sci 2006; 42: 47-54
20. Kimata $\mathrm{H}$. Enhancement of allergic skin wheal responses in patients with atopic eczema/dermatitis syndrome by playing video games or by frequently ringing mobile phone. Eur J Clin Invest 2003; 33: 513-7.

21. Kimata $\mathrm{H}$. Enhancement of allergic skin Wheel responses in patients with atopic eczema/dermatitis syndrome by playing video games or by frequently ringing mobile phone. Eur J Clin Invest 2003; 33: 513-7.

22. Ikezawa Z, Komori J, Ikezawa Y, et al. A role of Staphyococcus aureus, interleukin-18, nerve growth factor and semaphorin $3 \mathrm{~A}$, an axon guidance molecule, in pathogenesis and treatment of atopic dermatitis. Allergy Asthma Immunol Res 2010; 2: 235-46.

23. Ohshima M, Miyake M, Takeda M, et al. Staphylococcal Enterotoxin B causes proliferation of sensory C-fibers and subsequent enhancement of neurogenic inflammation in rat skin. J Infect Dis 2011; 203: 862-9.

24. Fantini F, Pincelli C, Romualdi $P$, et al. Substance $P$ levels are decreased in lesional skin of atopic dermatitis. Exp Dermatol 1992; 1: 127-8.

25. Giannetii A, Fantini F, Cimitan A, et al. Vasoactive intestinal polypeptide and substance $P$ in the pathogenesis of atopic dermatitis. Acta Derm Venereol Suppl 1992; 176: 90-2.

26. Giannetti A, Girolomoni G. Skin reactivity to neuropeptides in atopic dermatitis. Br J Dermatol 1989; 121: 681-8.

27. Heyer G. Abnormal cutaneous neurosensitivity in atopic skin. Acta Derm Venereol 1992; 176: 93-4.

28. Sugiura H, Omoto $M$, Sirota $Y$, et al. Density and fine structure of peripheral nerves in various skin lesions of atopic dermatitis. Arch Dermatol Res 1997; 289: 125-31.

29. Raap U, Werfel T, Goltz C, et al. Circulating levels of brainderived neurotrophic factor correlate with disease severity in the intrinsic type of atopic dermatitis. Allergy 2006; 61: 1416-8.

30. Negi O, Tominaga M, Tengara S, et al. Topically applied semaphorin 3A ointment inhibits scratching behavior and improves skin inflammation in NC/Nga mice with atopic dermatitis. J Dermatol Sci 2012; 66: 37-43.

31. Papoiu ADP, Wang $H$, Nattkemper L, et al. A study of serum concentrations and dermal levels of NGF in atopic dermatitis and healthy subjects. Neuropeptides 2011; 45: 417-22.

32. Umemoto N, Kakurai M, Okazaki H, et al. Serum levels of vasoactive intestinal peptide are elevated in patients with atopic dermatitis. J Dermatol Sci 2003; 31: 161-4.

33. Yamaguchi J, Michiko A, Kobayashi Y, et al. Quantitative analysis of nerve growth factor (NGF) in the atopic dermatitis and psoriasis horny layer and effect of treatment on NGF in atopic dermatitis. J Dermatol Sci 2009; 53: 48-54.

34. Peters EMJ, Liezmann C, Spatz K, et al. Nerve growth factor partially recovers inflamed skin from stress-induced worsening in allergic inflammation. J Invest Dermatol 2011; 131: 735-43.

35. Aloe L, Bracci-Laudiero L, Alleva E, et al. Emotional stress induced by parachute jumping enhances blood nerve growth factor levels and the distribution of nerve growth factor receptors in lymphocytes. Proc Natl Acad Sci USA 1994; 91: 10440-4.

36. Herberth G, Daegelmann C, Weber A. Association of neuropeptides with Th1/Th2 balance and allergic sensitization in children. Clin Exp Allergy 2006; 36: 1408-16.

37. Kaczmarski M, Wasilewska J, Jarocka-Cyrta E, et al. Polish statement on food allergy in children and adolescents. Postep Derm Alergol 2011; 28: 331-67.

38. Rosińska-Więckowicz A, Czarnecka-Operacz M. Disease extent and severity in patients with atopic dermatitis and food allergy. Postep Derm Alergol 2011; 28: 382-8. 
39. Nowacki Z. Patients characteristics of children with cow's milk allergy presenting with an allergy to extensively hydrolyzed whey and/or casein formulae used as part of an elimination diet. Postep Derm Alergol 2012; 29: 421-31.

40. Voice JK, Grinninger C, Kong Y, et al. Roles of vasoactive intestinal peptide (VIP) in the expression of different immune phenotypes by wild-type mice and T cell-targeted type II VIP receptor transgenic mice. J Immunol 2003; 170: 308-14.

41. Delgado M, Ganea D. Is vasoactive intestinal peptide a type 2 cytokine. J Immunol 2001; 166: 2907-12.

42. Kabashima-Kubo R, Nakamura M, Sakabe J, et al. A group of atopic dermatitis without IgE elevation or barrier impairment shows a high Th1 frequency: possible immunological state of the intrinsic type. J Dermatol Sci 2012; 67: 37-43. 